\title{
Los negocios realizados por internet, ¿cambian los paradigmas existentes?*
}

\author{
Business Performed on Internet Change \\ Existing Internet Porradigms
}

\section{Beatriz Marlene Rodríguez Acosta**}

\section{RESUMEN}

Internet en sus inicios fue una red de carácter militar restringida, que evolucionó hacia una red abierta pública y con acceso a casi todos los habitantes del planeta. Conforme a ello, este es un punto clave para que los negocios que se plantean como paradigmas tradicionales, cambien al usar esta herramienta, ya que está modificando los esquemas habituales de hacer las cosas. En este articulo se trata de determinar cómo han cambiado los negocios con la aparición de Internet, cómo han evolucionado.

\section{PALABRAS CLAVES}

Internet, paradigmas, negocios, evolución, relacionamiento.

\begin{abstract}
Internet in its beginnings was a restricted military network that evolved into an open public network with access to a Imost all peoples of the planet. In sight of that this is a key point for those businesses that consider themselves as traditional paradigms, this tool change the usual schemes of making things by modifying it. This article is about determining how businesses have changed with the appearance of the Internet and how they have evolved.
\end{abstract}

\section{KEYWORDS}

Internet, paradigms, business, evolution, relationship.

*Artículo recibido el 3 de julio de 2017 y aceptado el 3 de noviembre de 2017

${ }^{*}$ Capitulo Uruguay de la Sociedad de Internet (ISOCUY). (bemaroa@gmail.com) orcid 0000-0003-2572-2804 
SUM ARIO

1. Introducción

2. Paradigmas que se han modificado con internet

3. Cómo internet cambió la manera de hacer negocios

4. Evolución de los negocios en internet

5. Conclusiones

\section{Introducción}

Internet, desde sus comienzos, ha sido una herramienta para el cambio, de paradigmas fundamentales como el acceso, el espacio, el tiempo, el comercio, los negocios y la contratación, así como la seguridad y la privacidad.

¿Cómo han evolucionado estos cambios? ¿Cómo han influido en los negocios? Estas son preguntas que se originan como fondo de este tema.

Los seres humanos son sociales, por lo que necesitan convivir bajo normas comunes y confiar en lo que tienen a su alrededor y en sus pares. Así, comparten y cooperan entre sí. Ese es un fundamento básico para que los cambios realizados con la aplicación de internet los ayude a que los modelos tradicionales se modifiquen.

El vocablo paradigma, en un sentido amplio, significa algo que va a servir como modelo o ejemplo en una situación dada; son las directrices de un grupo que determinan cómo una persona debe actuar dentro de los límites.

Cuando se circunscribe al ámbito de la evolución y proceso de cambio paradigmático, hay que destacar, según Joel Baker, ${ }^{1}$ que un paradigma puede estar sujeto a evolución cuando, por el transcurrir del tiempo, sufre cambios para adaptarse a las nuevas realidades del mundo. Por evolución, un paradigma puede dar lugar a diversos paradigmas en su misma sintonía de pensamiento, $o$ sufrir transformaciones hasta que ya no sea aplicable a la realidad por lo cual muere. Con ello se gesta un nuevo paradigma o el cambio a cero.

Los negocios on line, es decir, aquellos que se ejecutan a través de canales o medios electrónicos, o por una página web, ${ }^{2}$ también han sido alcanzados por los cambios de paradigmas. Acorde con lo indicado precedentemente y realizando el estudio cualitativo de estos elementos, se considera que los negocios, con la llegada de internet, han sido susceptibles de modificaciones.

\footnotetext{
1 Barker, Joel, Paradigmas. El negocio de descubrir el futuro, Bogotá, Colombia, Mac Graw Hill, 1995.

2 Guzmán Rodriguez, Selvio, "Negocios on line", Negocios Online y Más. [Consulta: 25 de octubre, 2017]. Disponible en: https://negociosonlineymas.blogspot.com.uy/2013/11/concepto-de-negocios-online-la-tendencia.html [Consulta: 25 de octubre, 2017].
} 


\section{Paradigmas que se han modificado con internet}

Cuando un paradigma cambia, establece nuevas condiciones y supuestos que traen consigo retos, oportunidades y un cambio de pensamiento, dependiendo de la época en que se produce. El éxito de cada persona y organización dependerá del entendimiento y adaptación a estos. La teoría de la evolución sugiere que el mundo es de los que se adaptan mejor a él; sin embargo, cuando se llega al extremo de la especialización se corre el riesgo de que cuando el mundo cambie se esté condenado a desaparecer. ${ }^{3}$

Esta teoría está basada en la teoría biológica de Charles Darwin. Sólo las empresas que se adapten a un entorno cambiante van a sobrevivir. ${ }^{4}$ Esto sucede con el advenimiento de las nuevas tecnologías y la influencia que estas han tenido en los negocios.

Ellos, de acuerdo con lo señalado por Muñoz, ${ }^{5}$ establecen reglas de cómo se deben hacer las cosas, limitando a las personas a pensar en situaciones establecidas. Bloquean nuestra capacidad de observar el mundo como un todo y de buscar alternativas pensando de manera no convencional. Ello se aplica en todos los niveles, ya sea económico, social, político, entre otros. Surge cuando se acumula un exceso de cuestiones que se salen del marco ordinario y el paradigma actual no puede explicar. Además, implica un principio que siempre existió pero que hasta entonces nadie había reconocido: es incluyente.

Un nuevo paradigma exige dar vuelta e iniciar con una hoja en blanco. Es una transformación radical del significado de las reglas prevalecientes hasta esos momentos, y cuando explica lo que hasta entonces era turbio y oscuro, poco a poco va ganando terreno. Después de algún tiempo, este paradigma comienza a tener grietas y surge uno nuevo; a este proceso repetitivo se le llama evolución. ${ }^{6}$

Los modelos que han cambiado y que inciden en forma más profunda en los negocios son los conceptos de tiempo, espacio, acceso y comercio, gracias al advenimiento de las tecnologías, y en especial de internet. Se ha producido

\footnotetext{
${ }^{3}$ Muñoz Verdesoto, Fabián, "La nueva visión ecuatoriana", Monografías. [Consulta: 25 de octubre, 2017]. Disponible en: http://www.monografias.com/trabajos14/reingenieria-hum/reingenieria-hum.shtml

${ }^{4}$ De la PeÑa, José, "La teoría de la evolución y los negocios", Sandopen. [Consulta: 25 de octubre, 2017]. Disponible en: http://sandopen.blogspot.com.uy/2008/05/la-teora-de-la-evolucin-y-los-negocios.html

${ }^{5}$ Muñoz Verdesoto, Fabián, "La nueva visión ecuatoriana", Monografías. [Consulta: 25 de octubre, 2017]. Disponible en: http://www.monografias.com/trabajos14/reingenieria-hum/reingenieria-hum.shtml

${ }^{6}$ Muñoz Verdesoto, Fabián, "La nueva visión ecuatoriana", Monografías. [Consulta: 25 de octubre, 2017]. Disponible en: http://www.monografias.com/trabajos14/reingenieria-hum/reingenieria-hum.shtml
} 
la evolución que adapta a los individuos a los cambios, ${ }^{7}$ aunque existan otros cambios que influyen en menor grado que estos.

El tiempo actualmente es llamado tiempo real; es aquel en el que todo lo que se realiza por internet va a suceder en todas partes al mismo momento.

La palabra tiempo significa que el correcto funcionamiento del sistema depende no sólo del resultado lógico, sino también del tiempo en que se produce un cambio o un resultado. La palabra real, dentro de los sistemas, se refiere a la reacción que esta tiene a eventos externos que se realizan durante su funcionamiento.

En otras palabras, se puede decir tiempo real a recibir un dato o información en el menor tiempo posible.

Los datos en tiempo real en internet no necesariamente significan que la información está disponible en el momento en el que se recopila o en el momento en el que se envía, sino que es todo dato o información que se actualiza en forma regular y que cambia con frecuencia.

Las imágenes satelitales nuevas se actualizan cada hora. A esto se le conoce como datos en tiempo real. Pero los datos de temperatura recolectados desde 1972 hasta 1986 por medio de globos meteorológicos no se consideran datos en tiempo real, porque esos datos no cambian. ${ }^{8}$ En relación con este concepto, se habla de una historia presente, debido a la forma instantánea en la que se pueden producir los hechos.

Otro de los aspectos que se transforman con la red de redes es el de distancia, lo cual significa "que se realiza o funciona por correspondencia o a través de los medios de telecomunicación, sin que se requiera la presencia física de los participantes”. La noción de espacio no es la misma. Ya no existirán fronteras, y si una persona con la que estamos realizando un negocio está en la habitación de al lado o al otro lado del mundo, no importa, porque internet, a la que también llamamos autopista de la información, no se verá limitada por ninguna extensión.

Este concepto de fronteras sin fronteras determina que los negocios obtengan un público objetivo más amplio. Se entiende que se puede llegar a un público potencial, en forma indirecta, es decir, que se puede obtener productos de cualquier ofertante en cualquier parte del mundo. Ello es gracias a internet.

\footnotetext{
7 Sandoval, Frankun, "Internet: el arte de romper paradigmas", Monografías. [Consulta: 23 de junio, 2017]. Disponible en: http://www.monografias.com/trabajos/tesisinternet/tesisinternet.shtml

8 "¿Qué es tiempo real?", Esamuelm's Weblog. [Consuta: 24 de junio, 2017]. Disponible en: https://esamuelm.wordpress.com/2009/01/15/\%C2\%BFque-es-tiempo-real/
} 
En países como Uruguay, se entiende que este concepto está contenido en los negocios que se realizan por mensajero en los que, en relación con el artículo 1265 del Código Civil Uruguayo, "El contrato ajustado por mensajero o por correspondencia epistolar o telegráfica, se perfecciona en el lugar y en el acto en que la respuesta del que aceptó el negocio llega al proponente". ${ }^{9}$ Es decir, no es necesario que para su perfeccionamiento se encuentren en el mismo lugar el oferente y quien aceptó la oferta.

El acceso es el tercer paradigma modificado por la aparición de internet. Este nos lleva a que se tenga que cambiar la modalidad de pensamiento, a que ahora no se piense en estar solamente en la zona de confort, sino que se debe salir de ella, a pesar de no hacerlo físicamente.

Actualmente, el acceso determina que los negocios se puedan realizar en cualquier momento. No es necesario ir a un lugar específico y concreto; antes era inevitable que se realizara un encuentro entre las partes del negocio en forma personal.

En cuarto lugar, el concepto que se ha transformado es el de comercio. Este ha sido una actividad ancestral desde que la humanidad comprendió los beneficios que le brindaba hasta el día de hoy.

Con la aparición de la web, las tecnologías y las telecomunicaciones se desarrollan de forma tal que los intercambios de datos crecieron a niveles extraordinario. Se han simplificado cada vez más y han creado nuevas formas. En este marco se desarrolla el comercio electrónico. ${ }^{10}$

La peculiaridad de este nuevo comercio no radica en ser una forma diferente, sino que se lleva a cabo a través de medios distintos. Esto hace emerger una problemática jurídica diferente, así como una mayor cantidad de definiciones que van más allá del simple uso de internet como medio para realizar actividades comerciales. El rol que cada uno de estos modelos cumple en los negocios sufre una modificación al utilizar internet como una herramienta para su realización.

Con respecto a estos cambios de modelos, además de las preguntas que se han formulado al principio, surgen otras: ¿qué sucede con la unidad de acto?, ¿qué acontece si están las partes del contrato en diferentes lugares?, ¿las solemnidades necesarias en determinados negocios al realizar los contratos permanecen o no?, ¿se considera que la regulación es suficiente o se debe ampliar o modificar la ya existente?

\footnotetext{
${ }^{9}$ Código Civil Uruguayo, Normativa y Avisos Leglales del Uruguay. [Consulta: 26 de junio, 2017]. Disponible en: https://www.impo.com.uy/bases/codigo-civil/16603-1994

${ }^{10}$ Campitell, Adrián, "Comercio electrónico", Monografías. [Consulta: 15 de junio, 2017]. Disponible en: http://www. monografias.com/trabajos12/monogrr/monogrr.shtml
} 


\section{Cómo internet cambió la manera de hacer negocios}

Para Eduardo Santoyo, gerente general de “.CO”:

El cambio en la forma en la que hacemos negocios hoy en día es cada vez más evidente. No sólo tenemos en internet un vehículo para llegar a un mayor número de clientes potenciales -a quienes podemos presentarles al detalle nuestra oferta de productos y servicios- sino también es más fácil ahora introducir nuevas formas para lograr que la relación cliente-proveedor sea más sencilla y cómoda para ambas partes, precisamente por las nuevas posibilidades de e nvío de productos o servicios -y claro- por las variadas formas de pago que existen hoy en día en el mercado. ${ }^{11}$

Internet cambia el negocio tradicional por el negocio on line. Sentarse frente a un escritorio a establecer cuáles son las cláusulas que van a determinar los derechos y obligaciones de las partes, cuáles son las que van a determinar las diversas partes del contrato desde el momento de la oferta hasta la ejecución, ya sea de bienes o servicios, esa unidad de acto necesaria para que no se cambien las condiciones del contrato, ya no son lo principal. Ahora se puede estar en diferentes países, lugares, y realizar un negocio sin que haya perdido las características jurídicas que antes tenían.

Desde el momento en que se realiza la oferta hasta la ejecución del negocio se producen transformaciones. Inicialmente, el ofrecimiento se efectuaba a través de los medios de comunicación (diarios, revistas), o en forma oral (de boca en boca); empero, actualmente se realiza a través de internet, mediante las páginas web.

Como consecuencia de ello, las empresas y ofertantes debieron evolucionar en su pensamiento, para seguir estando en el mundo de los negocios. Estas transacciones pasaron a ser totalmente on line; otras, se han convertido en sistemas mixtos. Esto es, se ejecutan mediante la red de redes y fuera de ella. Un ejemplo es la compra de libros por internet. Se puede comprar on line pero recibirlo mediante el correo postal, es decir, off line.

Si bien se ha estado hablando de los diversos paradigmas, se necesita trabajar con ellos para contestar todas las interrogantes que se plantearon anteriormente:

\footnotetext{
11 "Cómo internet cambió la manera de hacer negocios", Enter. [Consulta: 16 de junio, 2017]. Disponible en: http:// www.enter.co/guias/lleva-tu-negocio-a-internet/como-internet-cambio-la-manera-de-hacer-negocios
} 
a) ¿Qué sucede con el concepto de unidad de acto?, ¿qué acontece si están las partes del contrato en diversos lugares?

El concepto de unidad de acto se ha dado en los negocios a través de los contratos desde que estos han surgido. Es esencial para comprender el instrumento público, aquél por el cual se realiza el negocio.

En relación con el tema, el notario Antonio Rodríguez Adrados indica que "La unidad del acto ha sido objeto de un fuerte rechazo, por incompatible con la agilidad del tráfico, siempre creciente". ${ }^{12}$

La unidad del acto supone audiencia notarial plena, dada por la presencia en el mismo espacio y tiempo de los sujetos del instrumento notarial en el acto de otorgamiento y autorización del documento público. ${ }^{13}$

Para Alberto Gaete, la contratación electrónica resulta estructuralmente diferente a la contratación clásica. Considera, además, que desaparece la unidad del acto entendida como unidad temporal y espacial propia de la expresión del consentimiento contractual, tanto material (que implica simultaneidad en la exteriorización de las voluntades) como formal, o simultaneidad entre las voluntades de las partes y aquella del autorizante. Asimismo, afirma que es de un doble carácter: en cuanto al acto, debe ser ininterrumpida; en su dimensión papel, debe estar contenida en un solo instrumento. Esta última, según el autor, constituye verdaderamente unidad de texto; es la que permanece en el documento electrónico. ${ }^{14}$

En un contexto electrónico, de intercambio de información digital entre las partes y el notario, sancionada y rubricada con sus respectivas claves y códigos algorítmicos, donde no medie contacto físico, únicamente se puede hablar, en algún sentido, de unidad del acto si, reformulando las dimensiones de espacio y tiempo, las partes y el notario se encuentran en red, interconectadas sus computadoras. Deben realizar todas las operaciones en tiempo real y debe verificarse dicho principio de unidad del acto en el ciberespacio, en el espacio de comunicación virtual, espacio racional donde los individuos conversan e intercambian datos por medio de terminales y redes entrelazadas.

\footnotetext{
12 Rodriguez Adrados, Antonio, "La unidad de acto formal", El notario del siglo xxı. [Consulta: 18 de junio, 2017]. Disponible en: http://www.elnotario.es/index.php/hemeroteca/revista-24/1666-principio-de-la-unidad-de-actoformal-0-12874558393726646

${ }^{13}$ Fomento ZAYAS, YAniXet, "Documento electrónico e instrumento público: la función del notario", Monografías. [Consulta: 18 de junio, 2017]. Disponible en: http://www.monografias.com/trabajos43/documento-electronico/documento-electronico2.shtml

${ }^{14}$ Gaete, Eugenio Alberto, "Documento electrónico e instrumento público", Portal de Abogados. [Consulta: 16 de junio, 2017]. Disponible en: http://www.portaldeabogados.com.ar/noticias/derin05.htm
} 
Sin embargo, sobre este punto aún queda mucho por debatir, sobre todo en aquellos contratos que están regidos por solemnidades, como las escrituras de compraventa de bienes inmuebles, que necesariamente se otorgan mediante escritura pública. Esta última debe utilizar todos los principios en su formulación, y el principio de unidad de acto debe mantenerse en forma primordial.

La utilización de las tecnologías en los negocios conduce a la posibilidad de administrar las relaciones entre clientes de manera mucho más personalizada, superando las barreras geográficas y obteniendo menores costos $\mathrm{y}$ tiempos.

b) ¿Las solemnidades necesarias en determinados negocios al realizar los contratos permanecen o no?

Los contratos que dan forma a los negocios en determinadas ocasiones cuentan con diversas solemnidades, como la escritura pública. Estos negocios están sujetos a la observancia de ciertas formalidades especiales. Sin ellas no produce ningún efecto civil. Por lo tanto, hasta el momento no se han podido realizar a través de internet, a pesar de la existencia en muchos países del concepto de documento y firma electrónica, los cuales tienen igual valor jurídico que el documento elaborado en papel y la firma ológrafa.

El cambio de los paradigmas, como resultado de la expansión de internet al llegar a los negocios, ha cambiado la forma de su ejecución. Por ello, se determina si la ley exige que se plasme el contrato en una forma definida para que sea válido y eficaz entre las partes y llegue a finalizar como debe ser, o si hay libertad de forma. En este último caso, el contrato se podrá finalizar sin las solemnidades necesarias.

Cada vez que se lleva a cabo un negocio se debe analizar si son necesarias las solemnidades y establecer si pueden realizarse mediante la libertad de forma, obligándose por el simple consentimiento de las partes sin las formalidades especiales regladas para los primeros. ${ }^{15}$

La normativa en general de contratación en el comercio está basada en el principio de libertad de forma, en el cual se perfecciona el negocio en el momento en que se produce el acuerdo de voluntades, sea cual fuere la forma elegida por las partes. No obstante, en países como España y Uruguay existen excepciones a este principio. Es el caso, por ejemplo, de la compraventa de

\footnotetext{
${ }^{15}$ GamarRa, Jorge, Tratado de derecho civil uruguayo, tomo 8, volumen 1, Montevideo, Fundación de Cultura Universitaria, 1979, p. 189.
} 
bienes inmuebles: es necesario cumplir con determinadas solemnidades, como la escritura pública.

c) ¿Cómo han evolucionado estos cambios?, ¿cómo han influido en los negocios?

Internet, al comienzo, era una red militar; luego se extendió a la academia, para salir más tarde al mundo en que los usuarios percibieron que se podía negociar utilizándola como herramienta.

Opera fundamentalmente como un facilitador de negocios, al decir de Luis del Barrio. Según el autor, ${ }^{16}$ el verdadero impacto de internet es que influye día a día sobre las empresas y las personas de múltiples maneras.

Esta herramienta y los cambios de paradigmas hacen posible que los negocios evolucionen y alcancen a una mayor cantidad de público, que sean más rápidos, que no se exista demora (debido a la burocracia), que el costo sea cada vez más reducido, que las formalidades y requisitos sean menos estrictos y que la efectividad sea real. Además, se vuelve necesario aceptar y proyectar las expectativas futuras, así como pronosticar el impacto que estos cambios realizan en los negocios.

Con esta evolución se produce un ciclo de realimentación que genera un cambio cualitativo en todo el proceso, lo cual habilita una innovación. Por este cambio, negocios que antes no eran considerados, debido a que era imposible encararlos, ahora pueden desarrollarse incluso en condiciones ventajosas.

A partir de la potencialidad de internet, se ha dado un cambio muy grande en la forma de concebir los negocios. Luis Joyanes ${ }^{17}$ expone claramente cuando plantea que la revolución de las tecnologías de la información y las comunicaciones está cambiando la cultura tradicional basada en un mundo material, al crear mundos virtuales en los cuales se puede buscar información, realizar negocios y hasta entretenerse.

Erik Tamplin, Jim Marchwick y Cortney Wanca ${ }^{18}$ realizan un análisis del impacto temprano de internet en los negocios. Se ha expandido rápidamente desde fines del anterior milenio, con la promesa de cambiar los negocios y la vida diaria. La vastedad de su alcance ha generado procesos de innovación muy complejos y, a su vez, ha capitalizado innovaciones y creado herramientas de amplio impacto.

\footnotetext{
${ }^{16}$ Del Barrio, Luis, Del business al e-business, Barcelona, Gestión 2000, 2003.

17 Joyanes, Luis, Cibersociedad, Madrid, McGraw Hill, 1997, p. 9.

${ }^{18}$ Tamplin, Erik, Marchwick, Jim Y Wanca, Cortney, "The Diffusion of Innvovation: The Fortune 100 and the Internet, COM 5305", Interactive Communication Research, Research Project-5305, 1997.
} 
d) ¿Implican estos cambios una nueva normativa o con la existente ya es suficiente?

Estos cambios alteran las reglas de juego para hacer negocios y abren una ventana de oportunidad a nuevos agentes. Nivelan posibilidades de desarrollo comercial entre organizaciones de envergaduras muy diferentes y generan mecanismos de intercambio que hacen desaparecer fronteras físicas. Así, se replantea el ciclo habitual de funcionamiento de los negocios en empresas o instituciones todavía no globalizadas.

Los cambios profundos en la forma de hacer negocios no pueden tratarse mediante el análisis de la complejidad estática, que se aprecia como si las partes fueran simplemente elementos de un rompecabezas. ${ }^{19}$

Lo indicado anteriormente lleva a la composición de la pregunta de este punto. La noción de negocio aparece aproximadamente en el siglo I después de Cristo, en un contexto y una época en la que no se tenía pensado que existiría la red de redes para influenciar en los cambios que en esta época se estaban realizando.

Consiguientemente, se dan diversas doctrinas que no son unánimes en cuanto a la influencia de internet y las nuevas tecnologías en los negocios; por lo tanto, se preguntan si se debe o no modificar sus cimientos.

Una primera posición establece que no sería necesario una nueva normativa, que los negocios se insertan en el concepto tradicional como tal, basado en una relación entre los individuos, con una unidad de acto y con formalidades solemnes o no, pero existentes.

Salvador Bergel entiende que existen referencias propias de los negocios de bienes y servicios, que no implican reconocer que ellos tienen una autonomía diferente a los demás negocios tradicionales. ${ }^{20}$ Sin embargo, otra parte de la doctrina piensa que los negocios han cambiado, que los modelos que los regían ya no son los que las normas existentes regulan y que cada vez entran más en un gran dinamismo.

Altmark $^{21}$ entiende que existe una moderna teoría de los negocios que se fundamenta en la aparición de nuevos paradigmas, nuevas instituciones y

\footnotetext{
${ }^{19}$ Petrella, Carlos, "Negocios electrónicos. Una puerta para el desarrollo del relacionamiento con los clientes", Finding. Disponible en: https://www.fing.edu.uy/catedras/disi/DISI/pdf/NEGOCIOS\%20ELECTRONICOS.pdf

${ }^{20}$ Bergel, Salvador, "Notas sobre la contratación informática", Revista de Derecho Privado y Comunitario, 1998, p. 180.

${ }^{21}$ Altmark, Daniel, La etapa precontractual de los contratos informáticos. Informática y derechos, aportes de doctrina internacional, Buenos Aires, Argentina, Depalma, 1987.
} 
nuevos principios. Se necesita hacer alguna adaptación para que estas nuevas formas de negocios se regulen.

A pesar que se han originado diversas posiciones doctrinarias, se entiende que la normativa existente en cuanto a los negocios, a través de sus contratos, es suficiente. Sólo se deberá proporcionar una nueva en aquellos casos en que no exista o no sea suficiente. Para sustentar la no regulación de los negocios on line, se considera que existen otros sistemas como las buenas prácticas y los códigos de conducta que logran una buena ordenación del negocio, fomentando la autorregulación.

El Diccionario de la Real Academia Española define autorregulación como la "acción y efecto de autorregularse", y autorregularse es el "hecho de regularse a sí mismo". Si continuamos desmembrando el vocablo llegamos a regular, una de cuyas acepciones es "determinar las reglas o normas a que debe ajustarse alguien o algo".

Este modelo de regular los negocios que usan como herramienta a las tecnologías presume la observancia de unas pautas de conducta - principios y normas éticas- cuyo cumplimiento previamente se ha fijado como objetivo. Simultáneamente, también constituye la expresión del compromiso de responsabilidad social de un determinado sector de la industria. Los ámbitos en los que la autodisciplina puede operar son ciertamente muy numerosos. Se pueden citar, entre otros, la publicidad, la contratación electrónica o el mercado de valores. $^{22}$

Los códigos de conducta están basados en reglas de buenas prácticas, las cuales poseen un alto nivel de seguridad jurídica, que los vuelve beneficiosos para las partes que componen el negocio. Estos últimos voluntariamente determinan cuáles son las obligaciones y derechos que este les proporciona.

\section{Evolución de los negocios en internet}

Luego del estudio de los cambios que han sufrido los negocios en relación con los cambios de paradigmas, es interesante saber cuál ha sido su evolución. Como consecuencia, se desarrollan diversas etapas: experimentación, asimilación de internet en la organización, reinvención, y transformación.

\footnotetext{
22 LóPEZ JIMÉnEZ, DAVID, "Los códigos de conducta como paradigmas de buenas prácticas: el caso del comercio electrónico", Encuentros Multidisciplinares. [Consulta: 25 de octubre, 2017]. Disponible en: http://www.encuentros-multidisciplinares.org/Revistan\%C2\%BA32/David_L\%C3\%B3pez_Jim\%C3\%A9nez.pdf
} 
En la primera etapa, la experimentación, el objetivo es estar en internet y empezar a experimentar con sus posibilidades. Para la realización de los negocios se emplean canales nuevos, como el correo electrónico; se utiliza un sitio web en el que se informa cuáles son sus requisitos para llegar a sus usuarios.

En la etapa dos, asimilación de internet en la organización, comienzan a integrarse procesos y sistemas de información con internet. Se empieza a verificar, por ejemplo, la ausencia del principio de unidad de acto, de la falta de solemnidades.

En la tercera fase, la reinvención, se inician los efectos de los cambios de paradigmas, se redefinen como consecuencia los modelos de negocios que se transforman en un hecho.

En la cuarta y última fase, llamada transformación, se produce una convergencia entre el negocio, tal y como ha funcionado desde siempre, y los efectos totales que producen los cambios de paradigmas. ${ }^{23}$

Las tecnologías de la información y la comunicación influyen en el estado actual de los negocios, en su forma de ejecutarse, en la interacción con los usuarios, y en el relacionamiento entre las partes interesadas. Estas son las herramientas que van a lograr que se cumplan las diversas etapas o fases mencionadas anteriormente.

Se pasa de tener un negocio que se presenta en forma estática a tener un negocio interactivo y similar al negocio de siempre. En estas etapas se busca el equivalente funcional con el establecimiento tradicional, el que también ha pasado por estas etapas, pero sin la aplicación de las tecnologías.

\section{Conclusiones}

Internet constituye una fuente de recursos de información y conocimiento compartido a escala mundial; es también la vía de comunicación que permite establecer la cooperación y colaboración entre gran número de comunidades y grupos de interés por temas específicos, distribuidos por todo el planeta. Internet es una palabra que está de moda y que está cambiando los esquemas tradicionales en las formas de comunicarse. Es además, una herramienta necesaria para nuestros negocios. ${ }^{24}$

\footnotetext{
${ }^{23}$ GonzÁlez, FaCundo, "Internet. Una nueva manera de hacer negocios", Monografías. [Consulta: 25 de junio, 2017]. Disponible en: http://www.monografias.com/trabajos88/internet-nueva-manera-hacer-negocios/internet-nueva-manera-hacer-negocios2.shtml

${ }^{24}$ Sandoval, FrankLin, "Internet: el arte de romper paradigmas", Monografias. [Consulta: 23 de junio, 2017]. Disponible en: http://www.monografias.com/trabajos/tesisinternet/tesisinternet.shtml
} 
Todos los análisis de tendencias de negocios on line parecen concluir que, cada vez más, internet será una pieza indispensable en los negocios, y que los cambios seguirán apareciendo y modificando lo que se conoce como comercio tradicional.

La reconversión y capacidad de adaptación, tanto a nivel individual como a nivel empresarial, se hacen más necesarias. Internet deja de ser un accesorio en el mundo empresarial para formar parte de la estructura básica de los negocios.

Cada vez que se plantee un negocio, se deben considerar todos los cambios de paradigma que se han suscitado con la aparición de la red de redes. Dichos cambios se han producido en los conceptos de tiempo, espacio, acceso y, en consecuencia, en la contratación y el comercio.

Es importante no olvidar que se logran diferentes beneficios como el menor costo de los trámites, la aceleración de éstos, las diversas formalidades y requisitos que ya no serán necesarios, y el acceso desde cualquier lugar, sin necesidad de estar en un punto fijo.

El negocio se ve, entonces, en una situación ventajosa al usar internet como herramienta para su evolución. La doctrina está dividida con respecto a si es necesario o no la promulgación de nueva normativa; no obstante, se entiende que lo mejor es adaptar la ya existente para las circunstancias que aún no están reguladas, y tomar como modelo la autorregulación a través de las buenas prácticas y códigos de conducta. 


\section{Bibliografía}

Altmark, DAniel, La etapa precontractual de los contratos informáticos. Informática y derechos, aportes de doctrina internacional, Buenos Aires, Argentina, Depalma, 1987.

BARKer, Joel, Paradigmas. El negocio de descubrir el futuro, Bogotá, Colombia, Mac Graw Hill, 1995.

Bergel, Salvador, "Notas sobre la contratación informática”, Revista de Derecho Privado y Comunitario, 1998.

CAmpitelli, Adrián, "Comercio electrónico", Monografías. [Consulta: 15 de junio, 2017]. Disponible en: http://www.monografias.com/trabajos12/monogrr/ monogrr.shtml

Código Civil Uruguayo, Normativa y Avisos Leglales del Uruguay. [Consulta: 26 de junio, 2017]. Disponible en: https://www.impo.com.uy/bases/codigo-civil/16603-1994

“Cómo internet cambió la manera de hacer negocios”, Enter. [Consulta: 16 de junio, 2017]. Disponible en: http://www.enter.co/guias/lleva-tu-negocio-a-internet/como-internet-cambio-la-manera-de-hacer-negocios

De la PeÑA, José, “La teoría de la evolución y los negocios”, Sandopen. [Consulta: 25 de octubre, 2017]. Disponible en: http://sandopen.blogspot.com. uy/2008/05/la-teora-de-la-evolucin-y-los-negocios.html

Del Barrio, Luis, Del business al e-business, Barcelona, Gestión 2000, 2003.

Fomento Zayas, YaniXet, "Documento electrónico e instrumento público: la función del notario”, Monografías. [Consulta: 18 de junio, 2017]. Disponible en: http://www.monografias.com/trabajos43/documento-electronico/documento-electronico2.shtml

Gaete, Eugenio Alberto, "Documento electrónico e instrumento público", Portal de Abogados. [Consulta: 16 de junio, 2017]. Disponible en: http://www.portaldeabogados.com.ar/noticias/derin05.htm

Gamarra, Jorge, Tratado de derecho civil uruguayo, tomo 8, volumen 1, Montevideo, Fundación de Cultura Universitaria, 1979.

GuZmÁn Rodríguez, Selvio, “Negocios on line”, Negocios Online y Más. [Consulta: 25 de octubre, 2017]. Disponible en: https://negociosonlineymas.blogspot.com. uy/2013/11/concepto-de-negocios-online-la-tendencia.html

GonZÁlez, FACUndo, "Internet. Una nueva manera de hacer negocios”, Monografías. [Consulta: 25 de junio, 2017]. Disponible en: http://www.monografias.com/ trabajos88/internet-nueva-manera-hacer-negocios/internet-nueva-manera-hacer-negocios2.shtml

JoYANEs, LuIs, Cibersociedad, Madrid, McGraw Hill, 1997.

LóPEZ JIMÉNEZ, DAVID, “Los códigos de conducta como paradigmas de buenas prácticas: 
el caso del comercio electrónico", Encuentros Multidisciplinares. [Consulta: 25 de octubre, 2017]. Disponible en: http://www.encuentros-multidisciplinares.org/Revistan\%C2\%BA32/David_L\%C3\%B3pez_Jim\%C3\%A9nez.pdf

MuÑoz Verdesoto, FABIÁn, “La nueva visión ecuatoriana”, Monografías. [Consulta: 25 de octubre, 2017]. Disponible en: http://www.monografias.com/trabajos14/ reingenieria-hum/reingenieria-hum.shtml

Petrella, Carlos, "Negocios electrónicos. Una puerta para el desarrollo del relacionamiento con los clientes", Finding. Disponible en: https://www.fing.edu. uy/catedras/disi/DISI/pdf/NEGOCIOS\%20ELECTRONICOS.pdf

“¿Qué es tiempo real?”, Esamuelm's Weblog. [Consuta: 24 de junio, 2017]. Disponible en: https://esamuelm.wordpress.com/2009/01/15/\%C2\%BFque-es-tiempo-real/

Rodríguez Adrados, Antonio, "La unidad de acto formal", El notario del siglo xxi. [Consulta: 18 de junio, 2017]. Disponible en: http://www.elnotario.es/index.php/hemeroteca/revista-24/1666-principio-de-la-unidad-de-acto-formal-0-12874558393726646

SANDOVAL, Franklin, "Internet: el arte de romper paradigmas", Monografías. [Consulta: 23 de junio, 2017]. Disponible en: http://www.monografias.com/trabajos/tesisinternet/tesisinternet.shtml

Tamplin, Erik, Marchwick, Jim y Wanca, Cortney, "The Diffusion of Innvovation: The Fortune 100 and the Internet, COM 5305", Interactive Communication Research, Research Project-5305, 1997. 